\title{
Potential biomarkers for antidiastole of tuberculous and malignant pleural effusion by proteome analysis
}

\author{
Jing Shi*,‡,1, Pu Li $i^{\ddagger} 2$, Lijin Zhou ${ }^{2}$, Suwen $\mathrm{Qi}^{3}$, Bo Wang ${ }^{2}$, Dandan $\mathrm{Li}^{2}$, Liang Duan², Wei \\ Xian Chen ${ }^{2}$, Jirong Xia ${ }^{1}$, Lin Zou ${ }^{1}$ \& Shuangshuang Yang ${ }^{1}$ \\ ${ }^{1}$ Department of Laboratory Medicine, the First Affiliated Hospital of Chongqing Medical University, Chongqing 400016, PR China \\ ${ }^{2}$ Department of Laboratory Medicine, the Second Affiliated Hospital of Chongqing Medical University, Chongqing 400010, PR \\ China \\ ${ }^{3}$ National-Regional Key Technology Engineering Laboratory for Medical Ultrasound, Guangdong Key Laboratory for Biomedical \\ Measurements \& Ultrasound Imaging, Department of Biomedical Engineering, School of Medicine, Shenzhen University, Shenzhen \\ 518060, PR China \\ *Author for correspondence: Tel.: +86 23 89012513; Fax: +86 23 89012513; sj_677@163.com \\ $¥$ Co-first authors and authors contributed equally
}

\begin{abstract}
Aim: To investigate novel potential biomarkers for antidiastole of tuberculous pleural effusion (TPE) from malignant pleural effusion (MPE). Materials \& methods: iTRAQTM-coupled LC-MS/MS were applied to analyze the proteome of TPE and MPE samples. The candidate proteins were verified by enzyme-linked immunosorbent assay. Results: A total of 432 differential proteins were identified. Enzyme-linked immunosorbent assay revealed significantly higher levels of fibronectin (FN) and cathepsin G (CTSG) in MPE than in TPE, but lower levels of leukotriene-A4 hydrolase (LTA4H). The receiver operator characteristic values were 0.285 for FN, 0.64 for LTA4H, 0.337 for CTSG and 0.793 for a combination of these candidate markers. Conclusion: FN, LTA4H and CTSG were identified as potential biomarkers to differentiate TPE from MPE and their combination exhibited higher diagnostic capacity.
\end{abstract}

First draft submitted: 7 June 2018; Accepted for publication: 4 December 2018; Published online:

22 February 2019

Keywords: cathepsin G • fibronectin $\bullet$ iTRAQTM • leukotriene-A4 hydrolase $\bullet$ lung cancer $\bullet$ malignant pleural effusion • potential biomarkers $\bullet$ quantitative proteomic analyze $\bullet$ tuberculosis $\bullet$ tuberculous pleural effusion

Tuberculosis (TB) is an infectious disease caused by the bacterium Mycobacterium tubercu losis (Mtb) [1] and affects the lung in the majority of patients [2]. There were an estimated 10.4 million new (incident) TB cases and 1.4 million deaths worldwide in 2015 [3]. Furthermore, TB remains one of the most frequent causes of pleural effusions attributing to antigenic proteins released by $M t b$. These antigen were permeated into the pleural cavity and trigger the delayed hypersensitivity reaction generating the accumulation of fluid in the cavity [1]. The detection of $M t b$ in the pleural fluid or pleural biopsy specimens is the gold standard for the diagnosis of tuberculous pleural effusion (TPE) [4-6]. However, both the microscopy of acid-fast bacilli and the culture of $M t b$ in the pleural fluid showed extremely weak sensitivities in the diagnosis of TB pleuritis $[1,7]$. Routine chemistry indexes could be observed but lack of specificity, stability and accuracy in the identification of TPE and malignant pleural effusion (MPE) [1]. Pleural fluid adenosine deaminase (ADA) is an important tool in the diagnosis of pleural TB. High ADA levels are related to the subset of activated $\mathrm{T}$ lymphocytes in response to tuberculous antigens [8]. And it was reported that pleural fluid ADA levels were also independent predictors of worse survivalin patients with MPE [9]. But the level of ADA may not have obvious antidiastole efficiency in antidiastole of TPE and MPE, although it performs great in the diagnosis of pleural TB.

In the present study, clinical samples of TPE and MPE collected from patients with lung cancer or TB pleuritis were used respectively for isobaric tagging for relative and absolute quantification (iTRAQTM)-based quantitative proteomic analysis. This study was expected to provide valuable novel biomarkers for the clinical antidiastole of TPE and MPE. In this study, iTRAQTM $[10,11]$ combined with liquid chromatography-tandem mass spectrometry

Future 8 Medicine 
Table 1. Laboratory characteristics of non-small-cell lung cancer and tuberculosis pleura effusions for proteome dataset.

\begin{tabular}{|lll|}
\hline Characteristics & NSCLC $(\mathbf{n}=\mathbf{2 0})$ & Tuberculous pleurisy $(\mathbf{n = 2 0})$ \\
\hline Males/females & $11 / 9$ & $7 / 13$ \\
\hline Age (years) & $50.56 \pm 6.52$ & $41.31 \pm 8.21$ \\
\hline ALB $(\mathrm{g} / \mathrm{l})$ & $42.80 \pm 2.85$ & $38.21 \pm 6.37$ \\
\hline ADA $(\mathrm{U} / \mathrm{l})$ & $21.89 \pm 2.69$ & $51 \pm 5.17$ \\
\hline LDH $(\mathrm{U} / \mathrm{l})$ & $245.26 \pm 13.45$ & $185.32 \pm 12.24$ \\
\hline CEA $(\mu \mathrm{g} / \mathrm{l})$ & $19.21 \pm 4.23$ & $8.21 \pm 3.26$ \\
\hline GLU $(\mathrm{mmol} / \mathrm{l})$ & $3.05 \pm 0.31$ & $2.98 \pm 1.06$ \\
\hline ADA: Adenosine deaminase; ALB: Albumin; CEA: Carcinoembryonic antigen; GLU: Glucose; LDH: Lactate dehydrogenase; NSCLC: Non-small-cell lung cancer.
\end{tabular}

ADA: Adenosine deaminase; ALB: Albumin; CEA: Carcinoembryonic antigen; GLU: Glucose; LDH: Lactate dehydrogenase; NSCLC: Non-small-cell lung cancer.

Table 2. Laboratory characteristics of four groups of pleural effusions for enzyme-linked immunosorbent assay analysis.

\begin{tabular}{|lllll|}
\hline Characteristics & Tuberculous pleurisy $(\mathrm{n}=40)$ & NSCLC $(\mathbf{n}=40)$ & PPEs $(\mathbf{n}=37)$ & Transudate $(\mathrm{n}=36)$ \\
\hline Males/females & $20 / 20$ & $18 / 22$ & $20 / 17$ & $17 / 19$ \\
\hline Age (years) & $54.14 \pm 19.05$ & $66.38 \pm 12.47$ & $54.38 \pm 17.44$ & $72.14 \pm 17.50$ \\
\hline ALB (g/l) & $22.96 \pm 6.44$ & $26.66 \pm 18.68$ & $16.26 \pm 10.52$ & $11.9 \pm 3.78$ \\
\hline GLU (mmol/l) & $5.75 \pm 2.66$ & $5.26 \pm 3.54$ & $5.51 \pm 2.86$ & $6.76 \pm 1.73$ \\
\hline LDH (U/l) & $370.82 \pm 254.44$ & $422.36 \pm 310.09$ & $407.46 \pm 540.03$ & $132.50 \pm 94.41$ \\
\hline ADA (U/l) & $44.73 \pm 25.51$ & $7.24 \pm 4.70$ & $17.77 \pm 29.93$ & $3.62 \pm 2.47$ \\
\hline
\end{tabular}

ADA: Adenosine deaminase; ALB: Albumin; GLU: Glucose; LDH: Lactate dehydrogenase; NSCLC: Non-small-cell lung cancer; PPE: Pleural effusions.

quantitation method, was applied to identify and quantify the differences of proteome in TPE and MPE clinical samples. And enzyme-linked immunosorbent assay (ELISA) was used for further verification.

\section{Materials \& methods}

Patient populations \& clinical specimens

A total of $20 \mathrm{TPE}$ and $20 \mathrm{MPE}$ specimens obtained from tuberculous pleurisy and non-small-cell lung cancer (NSCLC) patients were used to identify different expression proteins through proteomics approach. The samples were collected from the Department of Respiratory Medicine in the First Affiliated Hospital of Chongqing Medical University from November 2015 to August 2016. Tuberculous pleurisy was diagnosed according to the TB guidelines for the diagnosis and treatment of TB in the global TB report [12]. Patients with NSCLC were diagnosed according to the diagnosis and treatment guide of National Comprehensive Cancer Network [13], including four cases of stage I, six cases of stage II, 11 cases of stage III and nine cases of stage IV. The clinical characteristics of the patients were shown in Table 1.

To further investigate potential biomarkers from proteomic results in distinguishing TPE from MPE, 153 specimens were obtained from 40 patients with tuberculous pleurisy, 40 patients with NSCLC, 37 patients with parapneumonic pleural effusions (PPEs) and 36 patients with transudate of pleural cavity. The inclusion criteria of patients with PPEs were as follows: exudative effusions associated with bacterial pneumonia, lung abscesses or bronchiectasis; absence of Mtb in pleural fluid obtained from serial thoracentesis procedures; remission and recovery for $\geq 3$ months at follow-up visits after antibiotic treatment; and pathological manifestations of inflammatory pleuritis, chronic empyema or pleural fibrosis and plaques without evidence of Mtb.; the specimens were collected in the Second Affiliated Hospital of Chongqing Medical University from November 2016 to August 2017. Tuberculous pleurisy was diagnosed following the TB guidelines for the diagnosis and treatment of TB in the global TB report (2016) [14]. NSCLC patients were diagnosed according to the diagnosis and treatment guide of National Comprehensive Cancer Network [13]. The laboratory characteristics of these 153 specimens were summarized in Table 2.

The pulmonary TB patients coupled with lung cancer were excluded from this study. The patients with diabetes mellitus, nephritis, hepatitis and having any anticancer therapy were also excluded. $5 \mathrm{ml}$ of pleural effusions were collected in a sterile tube and centrifuged for $20 \mathrm{~min}$ at $4^{\circ} \mathrm{C}$ at the speed of $4000 \mathrm{rpm}$. The supernatant was then separated and stored at $-80^{\circ} \mathrm{C}$ for further analysis. Informed consent was received from all the patients before the specimens were collected. 
iTRAQ proteomics

Sample preparation for iTRAQ

Albumin and IgG in pleural effusion was removed by Proteo Prep Blue AlbuminandIgG Depletion kit (SigmaAldrich, MO, USA). $30 \mu \mathrm{l}$ pleural effusion (100 $\mu$ g protein) was mixed with $120 \mu \mathrm{l}$ deoxidizer reagent and then bathed at $37^{\circ} \mathrm{C}$ for $1 \mathrm{~h}$. Then $120 \mu \mathrm{l}$ alkylating agents were added and incubated for $10 \mathrm{~min}$ at room temperature in the dark. The sample was transferred to an ultrafiltration centrifugal tube and centrifuged at $4{ }^{\circ} \mathrm{C}, 11,500 \mathrm{rpm}$ for $30 \mathrm{~min}$ to remove the unreactive components. $150 \mu \mathrm{l}$ of tetraethylammonium bromide was added into each ultrafiltration device and then centrifuged at $4^{\circ} \mathrm{C}$ for $30 \mathrm{~min}$ at $11,500 \mathrm{rpm}$, repeated three-times and removed the discharge liquid, adding $100 \mu \mathrm{l}$ confected enzymatic hydrolysate into each tube and $37^{\circ} \mathrm{C}$ water bathing overnight. The protein sample digested by enzyme was centrifuged at room temperature for $10 \mathrm{~min}$ at $11,500 \mathrm{rpm}$. Then $50 \mu \mathrm{l}$ tetraethylammonium bromide was added and centrifuged for $5 \mathrm{~min}, 11,500 \mathrm{rpm}$ under room temperature. The supernatant was transferred into the centrifuge and total protein concentration was measured with BCA protein assay kit (Pierce Biotechnology, IL, USA).

\section{iTRAQ labeling}

Proteins $(100 \mu \mathrm{g})$ from each pool was blocked, digested and labeled in according to the iTRAQ protocol. The iTRAQ tags for MPE and TPE were 113 and 114, respectively. The labeled digests were combined into one sample mixture. To reduce any potential variation introduced by the labeling reaction, each sample was split in two identical aliquots of $50 \mu \mathrm{g}$ to perform two technical replicates with tag swapping. One microgram of each labeled sample was individually analyzed by LC-MS/MS to check the completeness of the labeling. Acquired data were searched with Sequest search engine, setting the iTRAQ labeling as variable modification.

\section{HPLC \& mass assay}

First dimensional separation at basic $P H$ reverse phase chromatography

Tryptic digest mixture was dissolved in $100 \mu \mathrm{l}$ mobile phase $\mathrm{A}(10 \mathrm{mM}$ ammonium hydroxide in water/acetonitrile, $98 / 2[\mathrm{v} / \mathrm{v}], \mathrm{PH}=10.5$ ) and was then injected via a 1200 series HPLC (Agilent) into a Zorbax Extend-C18 column $(150 \times 2.1 \mathrm{~mm}$, Agilent). The mobile phase B included $10 \mathrm{mM}$ ammonium hydroxide in water/acetonitrile, 10/90 $(\mathrm{v} / \mathrm{v})$, the linear gradient elution was performed with $5-45 \% \mathrm{~B}$ in $50 \mathrm{~min}$ at a flow rate of $0.3 \mathrm{ml} / \mathrm{min}$. A total of 12 fractions from the first dimensional separation was collected (one fraction per $4 \mathrm{~min}$ ) and then lyophilized for further second dimensional online LC-MS analysis.

\section{Reverse phase nanoflow HPLC \& tandem mass spectrometry}

The lyophilized digestion was re-dissolved in 2\% acetonitrile, $0.1 \%$ formic acid and loaded on ChromXP C18 nanoLC trap column. The online chromatography separation was employed on the Eksigent nanoLC-Ultra ${ }^{T M} 2 \mathrm{D}$ System. The trapping, desalting procedure was carried out at $2 \mu \mathrm{l} / \mathrm{min}$ for $10 \mathrm{~min}$ with $100 \%$ solvent A. Solvents were composed of water/acetonitrile/formic acid. Then, an elution gradient of $5-35 \%$ acetonitrile $(0.1 \%$ formic acid) in 50-min gradient was used on an analytical column. LC-MS/MS analysis was performed with a TripleTOF 5600 System fitted with a Nanospray III source. Data were acquired using an ion spray voltage of $2.5 \mathrm{kV}$, curtain gas of 30 PSI, nebulizer gas of 5 PSI and an interface heater temperature of $150^{\circ} \mathrm{C}$.

\section{Protein identification by LC-MS analysis}

The MS/MS data were analyzed for protein identification and quantification using ProteinPilot v.4.5 (AB Sciex, Inc., USA). The local false discovery rate was estimated with the integrated PSPEP tool in the ProteinPilot software after searching in uniprot human database, which contains 134,366 protein sequences downloaded from the website of UniprotKB Results (http://www.uniprot.org/uniprot/). Three upregulated proteins (cathepsin G [CG] and fibronectin [FN]) and one downregulated protein (leukotriene A-4 hydrolase [LTA4H]) were selected to perform further validation.

\section{ELISA validation}

The expression of CG, FN and LTA4H in human TPE and MPE were operated by sandwich ELISA kits purchased from R\&D systems (MN, USA). The protein levels were positively correlated with the depth of reaction product's color. According to the standard curve, the concentration of proteins in the pleural effusion were calculated. 
Table 3. The upregulated proteins $(n=15)$ and downregulated proteins $(n=15)$ for tuberculous pleural effusion compared with malignant pleural effusion.

\begin{tabular}{|c|c|c|c|}
\hline Accession number & Protein name & Ratio & ProtScore \\
\hline \multicolumn{4}{|c|}{ Downregulated proteins } \\
\hline sp|P06681 & Complement C2 & 10.67 & 4.28 \\
\hline $\operatorname{tr|B4DLJ9}$ & Biotinidase & 6.79 & 19.71 \\
\hline sp|P49908 & Selenoprotein P & 4.83 & 15.22 \\
\hline sp|P02751 & Fibronectin & 4.79 & 5.55 \\
\hline sp|P51884 & Lumican & 4.61 & 6.42 \\
\hline sp|P80108 & Phosphatidylinositol-glycan-specific phospholipase D & 4.37 & 52.04 \\
\hline sp|Q8WWA0 & Intelectin-1 precursor & 4.37 & 47.91 \\
\hline sp|P09211 & Glutathione S-transferase P & 4.33 & 4.91 \\
\hline sp|Q86UD1 & Out at first protein homolog & 4.29 & 16.01 \\
\hline sp|P02760 & Protein AMBP & 4.25 & 22.47 \\
\hline sp|P08311 & Cathepsin G & 4.25 & 10.93 \\
\hline sp|P40197 & Platelet glycoprotein V & 4.25 & 2.51 \\
\hline $\operatorname{tr} \mid \mathrm{C} 9 \mathrm{JF} 17$ & Thioredoxin-disufidereductase & 4.17 & 23.3 \\
\hline \multicolumn{4}{|c|}{ Upregulated proteins } \\
\hline $\operatorname{tr|HOYB57}$ & Pulmonary surfactant-associated protein B (fragment) & 0.40 & 8.1 \\
\hline sp|P05543 & Thyroxine-binding globulin & 0.40 & 2.11 \\
\hline sp|P29279 & Connective tissue growth factor & 0.37 & 3.23 \\
\hline sp|P01619 & Ig kappa chain V-III region 3-20 & 0.36 & 2.06 \\
\hline sp|P08294 & Extracellular superoxide dismutase [Cu-Zn] & 0.36 & 12 \\
\hline sp|P09960 & Leukotriene $\mathrm{A}-4$ hydrolase & 0.36 & 11.04 \\
\hline sp|P45974 & Ubiquitin carboxyl-terminal hydrolase 5 & 0.35 & 2.01 \\
\hline sp|P14543 & Nidogen-1 & 0.31 & 3.6 \\
\hline $\operatorname{tr|F8VPV9}$ & REVERSED ATP synthase subunit beta & 0.30 & 2.05 \\
\hline sp|Q9H939 & Proline-serine-threoninephosphatase-interacting protein 2 & 0.30 & 4 \\
\hline sp|P02743 & Serum amyloid P-component & 0.29 & 21.73 \\
\hline sp|P25789 & Proteasome subunit alpha type- 4 & 0.29 & 2 \\
\hline sp|P35443 & Thrombospondin-4 & 0.26 & 4 \\
\hline
\end{tabular}

Statistical analysis

All statistical calculations were performed using SPSS v16.0 (SPSS, Inc., IL, USA). Student's t-test was used to compare continuous variables. One-way ANOVA was employed to compare the variations among more than two groups. A two-tailed $\mathrm{p}$ value $\leq 0.05$ was considered statistically significant. Receiver operator characteristic (ROC) curves were used to present the diagnostic sensitivity, specificity and the areas under the ROC curves (AUC), according to Hanley and McNeil method [15]. Logistic regression analysis was applied to predict the diagnostic probability. All these tests were performed in triplicates.

\section{Results}

Differential proteins between TPE \& MPE

A total of 432 mapped proteins from the 14,221 iTRAQ-labeled peptides were identified though proteomic analysis using a cut-off of ProtScore $>1.3$ and CI $>95 \%(\mathrm{p}<0.05)$. According to the criteria of fold change $>2.0$ or $<0.5$ between TPE and MPE, we identified 219 downregulated and 76 upregulated differential expression proteins. Table 3 displays the most significantly expressed upregulated $(n=15)$ and downregulated proteins $(n=15)$ for differentiation of TPE from MPE. 


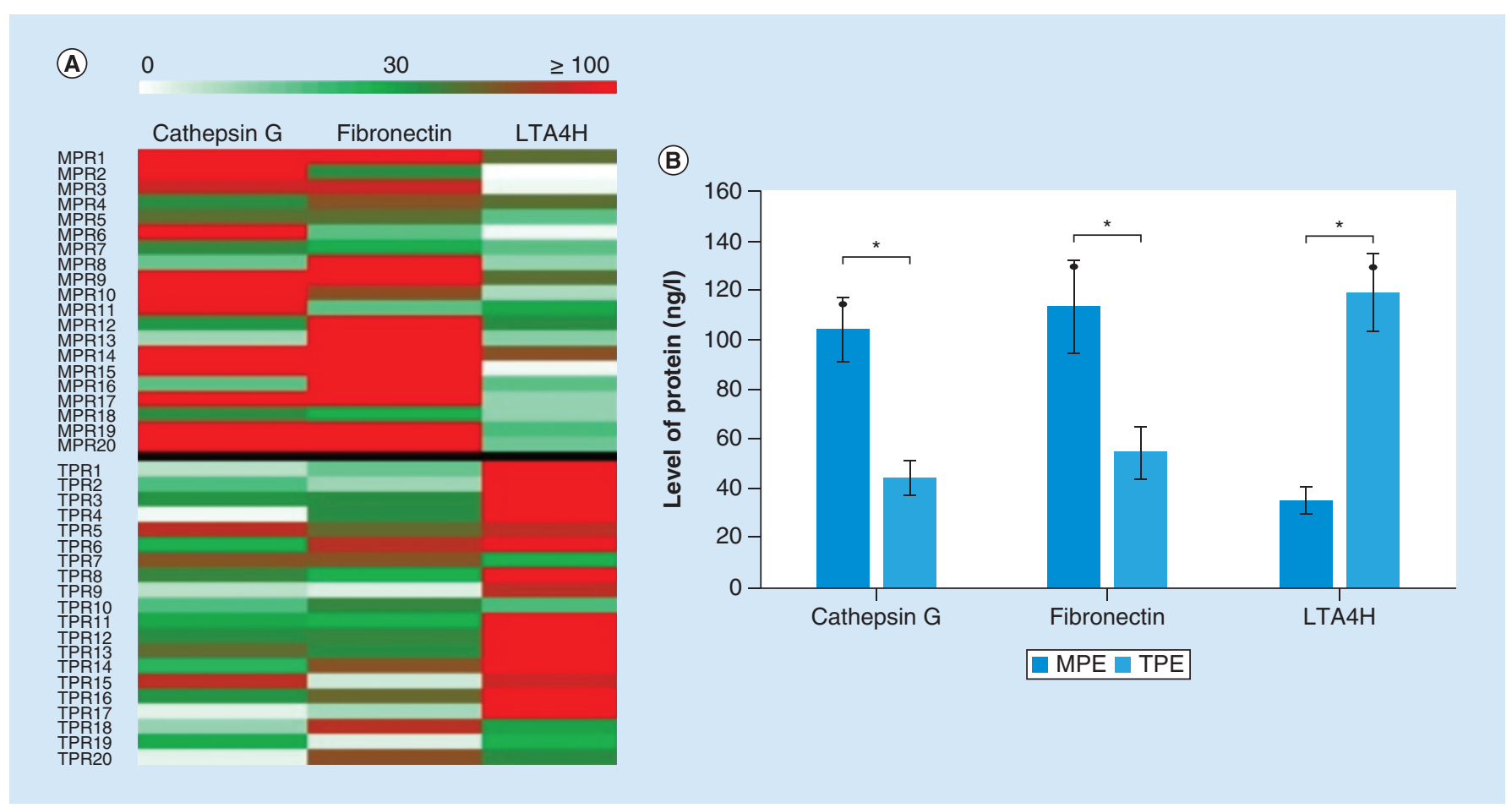

Figure 1. Three potential pleural effusion biomarkers for antidiastole of tuberculous pleural effusion and malignant pleural effusion. (A) Heat map of cathepsin, fibronectin and LTA4H levels in TPE and MPE samples. The heat map above the black line is the MPE group and the heat map under the black line is the TPE group. (B) The average levels of cathepsin, fibronectin and LTA4H expressed in TPE and MPE measured by proteomics analysis. The reported values are the mean \pm SD.

$* p<0.05$.

MPE: Malignant pleural effusion; SD: Standard deviation; TPE: Tuberculous pleural effusion.

For color figures please see online at: www.futuremedicine.com/doi/full/10.2217/bmm-2018-0200

\section{Three potential pleural effusion biomarkers for antidiastole of TPE \& MPE}

Among the most differentially expressed proteins, based on previous research, CTSG, FN and LTA4H were selected as candidates among the 30 differential proteins for further verification. Figure 1A presents the expressions of CTSG, FN and LTA4H in the TPE and MPE groups, stratified by the level of the TPE group. Combined with the average levels measured by proteomics analysis (Figure 1B), both CTSG and FN were higher in the MPE group than that in the TPE group, while the level of LTA4H expression was higher in the TPE group.

\section{Validation of pleural effusion biomarkers for distinguishing TPE \& MPE}

To verify the result of proteomics analysis, we extended the pleural effusion (PE) sample size to 153 specimens, comprising a TPE group $(\mathrm{n}=40)$, an MPE group $(\mathrm{n}=40)$, a PPEs group $(\mathrm{n}=37)$ and a 'transudate' group $(\mathrm{n}=36)$. ELISA was employed to perform preliminary clinical verification of the three candidates selected from proteomic analysis (CTSG, FN and LTA4H), as described above. As shown in Figure 2A, significant difference in the expression of FN was observed between the TPE and MPE groups. The level of FN was much higher in the MPE group than that in the TPE group $(\mathrm{p}<0.05)$, while compared with the PPEs and transudate groups, the FN in the TPE group was expressed in higher levels $(\mathrm{p}<0.05)$. Figure $2 \mathrm{~B}$ reveals particularly significant elevation of LTA4H in the TPE group, compared with other groups $(\mathrm{p}<0.05)$. The concentration of LTA4H in the TPE group was obviously higher than that in the MPE group $(\mathrm{p}<0.05)$. In Figure $2 \mathrm{C}$, a trend toward a higher CTSG expression was additionally observed in the MPE group. The level of CTSG in the TPE group was significantly lower than that in the MPE group ( $p<0.05)$; however, it was similar to that in the PPEs group $(p>0.05)$. The results revealed that the outcomes of ELISA were consistent with the proteomic analysis. Moreover, it had profound implications in distinguishing TPE and MPE that the levels of three potential pleural effusion biomarkers had evident statistical differences between the TPE and MPE groups. 


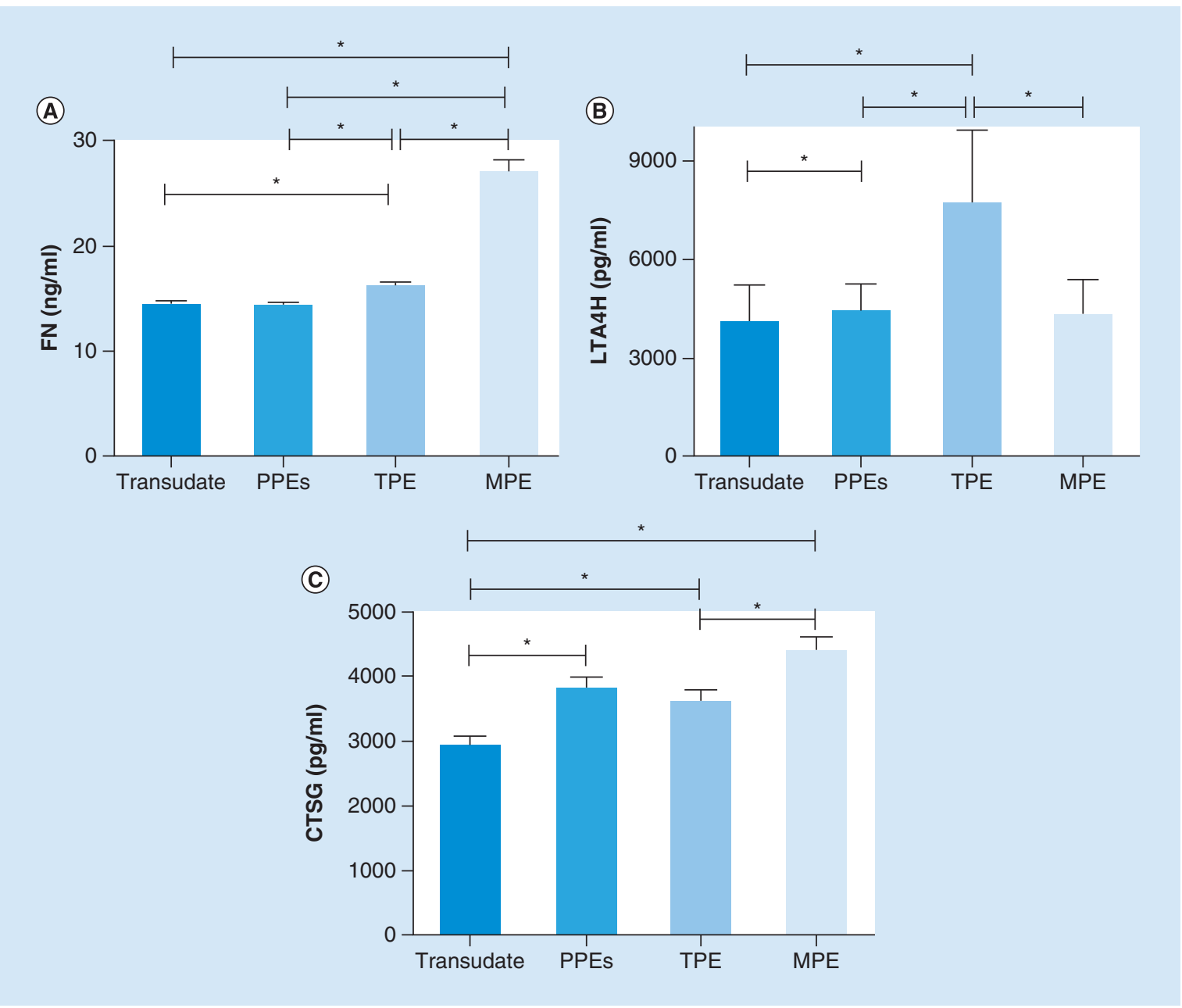

Figure 2. Detection of fibronectin, leukotriene-A4 hydrolase and cathepsin $\mathrm{G}$ in pleural effusions validated by ELISA. The concentrations of FN (A), LTA4H (B) and CTSG (C) in four different groups of PE analyzed by ELISA. Each column represents the mean \pm SD of at least three independent experiments.

${ }^{*} \mathrm{p}<0.05$.

CTSG: Cathepsin G; FN: Fibronectin; MPE: Malignant pleural effusion; PPE: Pleural effusions; SD: Standard deviation; TPE; Tuberculous pleural effusion.

\section{ROC analysis of pleural effusion biomarkers for the distinction of TPE \& MPE}

Based on the preliminary validation of the three candidate markers in different groups by ELISA, Figure 3 further displays the ROC plots of these markers and their combination for discrimination between TPE and MPE. The AUC value was determined to be 0.285 for FN (95\% CI: $0.174-0.396)$, 0.64 for LTA4H (95\% CI: $0.518-0.762$ ), 0.337 for cathepsin G (CTSG; 95\% CI: 0.218-0.456) and 0.793 for a combination of these candidate markers (95\% CI, 0.697-0.888). The highest predicate probability of the candidate markers combination measured via logistic regression analysis was 0.963 at the cut-off value of 13.37 (FN, ng/l), 8746.59 (LTA4H, pg/l) and 2070.91 (CTSG, pg/l) for distinction of TPE from MPE. Besides, the optimal sensitivity and specificity of the combination of all the indexes were 0.650 and 0.775 , respectively. Based on these results, the combination of three biomarkers exhibited higher antidiastole ability, relative to the three markers alone.

\section{Discussion}

TPE is the most common clinical manifestation of extra-pulmonary TB, which is one of the most frequent causes of pleural effusion [16,17]. Besides pulmonary TB, lung cancer is also prone to be complicated with PE [18-20]. The major challenges in laboratory medicine are related to the diagnosis and antidiastole of TPE with other types of pleural effusion. Our study applied proteomic technology to analyze clinical samples and reveal potentially 


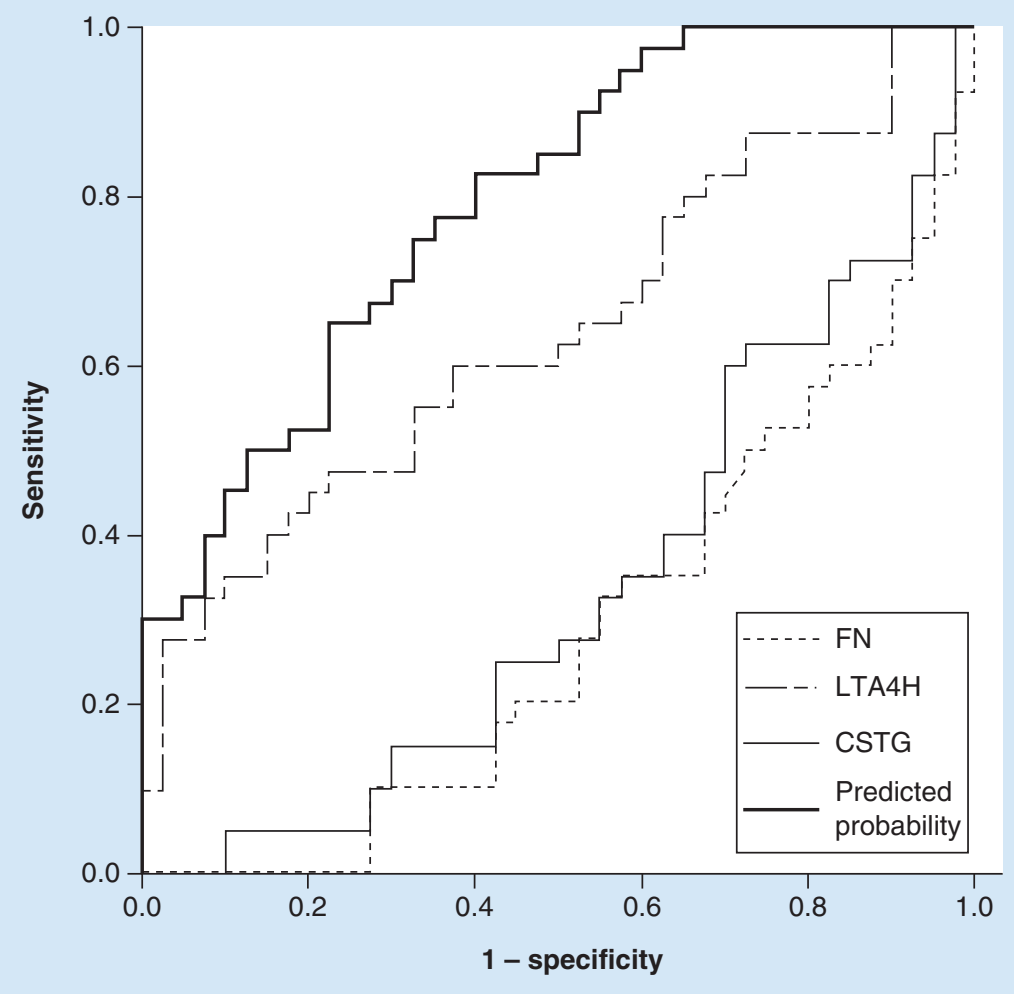

Figure 3. Reciever operating characteristic curves analysis of the three candidate markers. Based on the results of ELISA, reciever operating characteristiccurve analysis was employed to calculate the sensitivity, specificity and the areas under the curves (AUC). The predicate probability was 0.963 measured by logistic regression analysis. ROC analysis of FN, LTA4H, CTSG and a combination of three candidate markers for discrimination between tuberculous pleural effusion and malignant pleural effusion. The AUC value was determined as 0.285 for FN (95\% Cl: 0.174-0.396), 0.64 for LTA4H (95\% Cl: $0.518-0.762), 0.337$ for CTSG (95\% Cl: $0.218-0.456)$ and 0.793 for a combination of these candidate markers $(95 \% \mathrm{Cl}: 0.697-0.888)$. The optimal sensitivity and specificity of the combination of all the indexes were 0.650 and 0.775 , respectively.

CTSG: Cathepsin G; FN: Fibronectin.

meaningful biomarkers for antidiastole of TPE from MPE. This new study attempted to fill the gap in this area which has previously been neglected.

Nowadays, the definitive diagnosis of TPE depends on the demonstration of tuberculous bacilli in sputum, PE or pleural biopsy specimens or the observation of granulomas [21]. However, there exists a frequency of overlooks in sputum culture test for $M t b$ for the diagnosis of PE. The positive rate of $M t b$ culture is between 41.7 and $52 \%$ and it requires several weeks; however, the test offers $100 \%$ diagnostic specificity [22,23]. The detection of ADA is more common in TPE based on its high sensitivity and specificity [24,25]. Beside, the measurement of unstimulated pleural fluid IFN- $\gamma$ concentration by ELISA assay is also considered as a useful diagnostic tool for TB pleuritis [26]. While ADA remains an inflammatory biomarker, it confers a lack of specificity as the level of ADA significantly changes not only in TPE but also in MPE and is associated with worse survival of patients with MPE [9] The IFN- $\gamma$ test is not yet widely available in clinical practice and important information on its utility in low TB burden settings is also not yet available $[1,27]$. Furthermore, the determination of other routine chemistry indexes for the diagnosis of TPE has a lower yield than that of ADA and IFN- $\gamma$ and had these own localization [17]. Appropriate novel biomarkers need to be discovered to identify TPEs from other types of effusion.

Among the different pathogenic factors that induce MPE, lung cancer is the predominant etiology [28]. NSCLC accounts for $85 \%$ of all lung cancers, with the majority of patients initially diagnosed at advanced stages [29]. Furthermore, MPE and TPE contain plenty of protein components that directly relate to malignant tumor and tuberculous pleurisy. MPE is usually diagnosed by exfoliated cytological examination or transcutaneous pleural biopsy [30]. However, a limited number (only 40-70\%) of patients with MPE can be diagnosed via cytological 
examination or pleural biopsy [31]. To date, several potential biomarkers, carcinoembryonic antigen (CEA), lung surfactant protein A and VEGF, have been evaluated in MPE. However, due to limitations in sensitivity and specificity, these markers are not currently recommended or encouraged in routine clinical practice [30,32]. Therefore, the differentiation of TPE from MPE has profound implications in the diagnosis as well as therapy of TB and lung cancer.

To provide valuable novel biomarkers for the clinical antidiastole of TPE and MPE, isobaric tagging for relative and absolute protein quantification (iTRAQTM), combined with LC-MS/MS, was applied in this project [12,13]. We defined 432 different expression proteins from 20 PE clinical specimens of TPE and MPE (NSCLC). These contained 219 upregulating proteins and 75 downregulating proteins g. The 15 proteins with most significant differences in upregulation and downregulation are shown in Table 3. Based on the literature search, FN, LTA4H and CTSG were selected as the candidates among 30 different expression proteins for further verification. Finally, we discovered that both FN and CTSG had higher levels in the MPE samples compared to those in the TPE samples, while the LTA4H level in the TPE samples was significantly higher than that in the MPE samples.

FN, a member of the glycoprotein family with high-molecular weight and ligands of integrins, plays a major role in cell adhesion, growth, migration and differentiation, and it is also important for biological processes, such as wound healing and embryonic development [33-35]. Altered FN expression, degradation and organization have been associated with a number of pathologies, including cancer and fibrosis [33]. In addition, FN has also been reported as an important regulator of cisplatin-based chemotherapy resistance in advanced NSCLC [36]. LTA4H, a bio-functional enzyme with epoxy hydrolase and aminopeptidase activities, plays an important role as a chemoattractant for neutrophils [37]. Besides, it has been reported that combined drug therapy can ameliorate acute lung injury in mice models [38]. LTA4H also possesses aminopeptidase activity with the neutrophil chemoattractant proline-glycine-proline, which is a biomarker for chronic obstructive pulmonary disease [39]. A paucity of studies report the significance of elevated LTA4H in TPE. Hence, we speculate that the chronic inflammation associated with pulmonary TB may induce an accumulation of neutrophils and lead to the increase in LTA4H found in TPE. CTSG, a member of the peptidase S1 protein family, participates in the killing and digestion of engulfed pathogens and in connective tissue remodeling at inflammation sites [40,41]. In addition, CTSG and neutrophil elastase have been reported to play important roles in the deceleration of pathogen replication during the early phase of antimycobacterial responses [42] and were downregulated in THP-1 monocytes after infection with Mtb [43].

Furthermore, we collected more clinical samples to verify the proteomics results and the results of ELISA were consistent with those of proteomic analysis. Moreover, the PPEs group and transudate group were taken into consideration. Different expressions of candidates were not only found between TPE and MPE groups, but also existed among the other groups. This indicated that the three candidates were expressed at different levels in different conditions. Three potential biomarker levels in TPE were significantly different among other three groups. However, the CTSG level was similarly expressed in the TPE and PPEs groups. The underlying mechanisms deserve more investigation. Through the analysis of ROC curve, we found that LTA4H had a higher AUC value than CTSG and FN. Indeed, the potential biomarkers demonstrated an unexpected diagnostic efficiency, respectively, while their combination showed greater diagnostic yield. The combination of the three biomarkers exhibited higher diagnostic capacity, relative to the three markers alone (Figure 3).

\section{Conclusion}

In conclusion, FN, LTA4H and CTSG were identified as potential biomarkers to antidiastole TPE from MPE. Furthermore, their combination exhibited higher diagnostic capacity. These novel biomarkers could have a profound impact on the diagnosis and therapy of $\mathrm{TB}$ and lung cancer.

Financial \& competing interests disclosure

This work was supported by Chongqing Science \&Technology commission (No. cstc2015jcyjA10079) and Chongqing Municipal Education Commisson. (No. KJ1702036); the Project of the National Science Foundation of China under Grants 61401285, 81702083 and 81401750; Shenzhen Science Plan JCYJ20140418182819179. The authors have no other relevant affiliations or financial involvement with any organization or entity with a financial interest in or financial conflict with the subject matter or materials discussed in the manuscript apart from those disclosed.

No writing assistance was utilized in the production of this manuscript. 


\section{Ethical conduct of research}

The authors state that they have obtained appropriate institutional review board approval or have followed the principles outlined in the Declaration of Helsinki for all human or animal experimental investigations. In addition, for investigations involving human subjects, informed consent has been obtained from the participants involved.

\section{Acknowledgements}

The manuscript has been seen and approved by all authors. There is no conflict of interest for authors.

\section{Author's contributions}

All authors fulfill all of the four criteria:

- Substantial contributions to the conception or design of the work; or the acquisition, analysis, or interpretation of data for the work;

- Drafting the work or revising it critically for important intellectual content;

- Final approval of the version to be published;

- Agreement to be accountable for all aspects of the work in ensuring that questions related to the accuracy or integrity of any part of the work are appropriately investigated and resolved.

\section{Open access}

This work is licensed under the Attribution-NonCommercial-NoDerivatives 4.0 Unported License. To view a copy of this license, visit http://creativecommons.org/licenses/by-nc-nd/4.0/

\section{Summary points}

- An appropriate novel biomarker crucially needs to be discovered to identify tuberculous pleural effusions (TPEs) from other types of effusion.

- This study aimed to investigate novel potential biomarkers for antidiastole TPE from malignant pleural effusion (MPE).

Methods

- Quantitative proteomic analysis of the proteomes of TPE and MPE with iTRAQTM-coupled LC-MS/MS was performed.

Results

- The expressions of CTSG, FN and LTA4H were significantly different in TPE compared with those in para-pneumonic pleural effusions and transudate groups.

- FN, LTA4H and CTSG were identified as novel biomarkers for antidiastole TPE from MPE.

- The combination of FN, LTA4H and CTSG exhibited a higher diagnostic capacity.

Discussion

- This work provides valuable novel biomarkers for the clinical antidiastole of TPE from MPE.

- The identification of these biomarkers may confer a profound impact on the diagnosis and therapy of tuberculosis and lung cancer.

\section{References}

Papers of special note have been highlighted as: $\bullet$ of interest; $\bullet \bullet$ of considerable interest

1. Vorster MJ, Allwood BW, Diacon AH, Koegelenberg CF. Tuberculous pleural effusions: advances and controversies. J. Thorac. Dis. 7(6), 981-991 (2015).

- A key article about the advances and controversies of the research on tuberculous pleural effusions.

2. Porcel JM. Tuberculous pleural effusion. Lung 187(5), 263-270 (2009).

3. World Health Organization. Global tuberculosis report 2016. http://www.tbonline.info/posts/2016/10/16/global-tb-report-2016/

4. Diacon AH, Van De Wal BW, Wyser C et al. Diagnostic tools in tuberculous pleurisy: a direct comparative study. Eur. Respir. J. 22(4), 589-591 (2003).

5. Light RW. Update on tuberculous pleural effusion. Respirology 15(3), 451-458 (2010).

6. Koegelenberg CF, Bolliger CT, Theron J et al. Direct comparison of the diagnostic yield of ultrasound-assisted Abrams and Tru-Cut needle biopsies for pleural tuberculosis. Thorax 65(10), 857-862 (2010).

7. Valdes L, San-Jose E, Ferreiro L et al. Predicting malignant and tuberculous pleural effusions through demographics and pleural fluid analysis of patients. Clin. Respir. J. 9(2), 203-213 (2015). 
8. Yildirim H, Metintas M, Ak G et al. Increased pleural fluid adenosine deaminase levels in patients with malignant pleural effusions: a potential predictor of talc pleurodesis outcome. Lung 185(6), 349-354 (2007).

9. Terra RM, Antonangelo L, Mariani AW, De Oliveira RL, Teixeira LR, Pego-Fernandes PM. Pleural fluid adenosine deaminase (ADA) predicts survival in patients with malignant pleural effusion. Lung 194(4), 681-686 (2016).

10. Yang $\mathrm{Y}, \mathrm{Mu}$ J, Chen $\mathrm{G}$ et al. iTRAQ-based quantitative proteomic analysis of cerebrospinal fluid reveals NELL2 as a potential diagnostic biomarker of tuberculous meningitis. Int. J. Mol. Med. 35(5), 1323-1332 (2015).

-. An important article demonstrating iTRAQ-based quantitative proteomic analysis of CSF NELL2 is a potential diagnostic biomarker of tuberculous meningitis.

11. Ding Y, Yang M, She S et al. iTRAQ-based quantitative proteomic analysis of cervical cancer. Int. J. Oncol. 46(4), 1748-1758 (2015).

- An important article about iTRAQ-based quantitative proteomic analysis.

12. WHO. Treatment of tuberculosis guidelines for national programmes (2015). http://apps.who.int/bookorders/anglais/detart1.jsp?sesslan=1\&codlan=1\&codcol=15\&codcch=4397

13. Thurnher MM. 2007 World Health Organization classification of tumours of the central nervous system. Cancer Imaging 9(Spec No A), S1-S3 (2009).

14. WHO. Treatment guidelines for drug-resistant tuberculosis (2016). https://www.who.int/tb/areas-of-work/drug-resistant-tb/treatment/resources/en/

15. Hanley JA, Mcneil BJ. A method of comparing the areas under receiver operating characteristic curves derived from the same cases. Radiology 148(3), 839-843 (1983).

16. Zeng N, Wan C, Qin J et al. Diagnostic value of interleukins for tuberculous pleural effusion: a systematic review and meta-analysis. BMC Pulm. Med. 17(1), 180 (2017).

17. Ferreiro L, San Jose E, Valdes L. Tuberculous pleural effusion. Arch. Bronconeumol. 50(10), 435-443 (2014).

-. Demonstrates tuberculous pleural effusion is the most common form of extrapulmonary tuberculosis (TB), and is one of the most frequent causes of pleural effusion.

18. Rodriguez-Panadero F, Romero-Romero B. Management of malignant pleural effusions. Curr. Opin. Pulm. Med. 17(4), 269-273 (2011).

19. Simpson G, Judge DJ. Management of malignant pleural effusion. Respirology 20(1), 169 (2015).

20. Srour N, Amjadi K, Forster A, Aaron S. Management of malignant pleural effusions with indwelling pleural catheters or talc pleurodesis. Can. Respir. J. 20(2), 106-110 (2013).

21. Gopi A, Madhavan SM, Sharma SK, Sahn SA. Diagnosis and treatment of tuberculous pleural effusion in 2006. Chest 131(3), 880-889 (2007).

22. Valdes L, Ferreiro L, Cruz-Ferro E et al. Recent epidemiological trends in tuberculous pleural effusion in Galicia, Spain. Eur. J. Intern. Med. 23(8), 727-732 (2012).

23. Ruan SY, Chuang YC, Wang JY et al. Revisiting tuberculous pleurisy: pleural fluid characteristics and diagnostic yield of mycobacterial culture in an endemic area. Thorax 67(9), 822-827 (2012).

24. Qiu L, Teeter LD, Liu Z et al. Diagnostic associations between pleural and pulmonary tuberculosis. J. Inf. Secur. 53(6), 377-386 (2006).

25. Liang QL, Shi HZ, Wang K, Qin SM, Qin XJ. Diagnostic accuracy of adenosine deaminase in tuberculous pleurisy: a meta-analysis. Respir. Med. 102(5), 744-754 (2008).

26. Yurt S, Kucukergin C, Yigitbas BA, Seckin S, Tigin HC, Kosar AF. Diagnostic utility of serum and pleural levels of adenosine deaminase 1-2, and interferon-gamma in the diagnosis of pleural tuberculosis. Multidiscip. Respir. Med. 9(1), 12 (2014).

27. Dheda K, Van Zyl-Smit RN, Sechi LA et al. Utility of quantitative T-cell responses versus unstimulated interferon-\{gamma $\}$ for the diagnosis of pleural tuberculosis. Eur. Respir. J. 34(5), 1118-1126 (2009).

28. Heffner JE, Klein JS. Recent advances in the diagnosis and management of malignant pleural effusions. Mayo. Clin. Proc. 83(2), 235-250 (2008).

- Reports that among the different pathogenic factors induced malignant pleural effusion, lung cancer is the predominant etiology.

29. Moro-Sibilot D, Smit E, De Castro Carpeno J et al. Outcomes and resource use of non-small cell lung cancer (NSCLC) patients treated with first-line platinum-based chemotherapy across Europe: FRAME prospective observational study. Lung Cancer 88(2), 215-222 (2015).

30. Yu CJ, Wang CL, Wang CI et al. Comprehensive proteome analysis of malignant pleural effusion for lung cancer biomarker discovery by using multidimensional protein identification technology. J. Proteome Res. 10(10), 4671-4682 (2011).

31. Sallach SM, Sallach JA, Vasquez E, Schultz L, Kvale P. Volume of pleural fluid required for diagnosis of pleural malignancy. Chest 122(6), 1913-1917 (2002).

32. Domej W, Tilz GP, Földes-Papp Z et al. Cystatin C of pleural effusion as a novel diagnostic aid inpleural diseases of different aetiologies. Clin. Sci. (London) 102(3), 373-380 (2002). 
33. Malik G, Knowles LM, Dhir R et al. Plasma fibronectin promotes lung metastasis by contributions to fibrin clots and tumor cell invasion. Cancer Res. 70(11), 4327-4334 (2010).

- Describes that altered FN expression, degradation and organization have been associated with a number of pathologies, including cancer and fibrosis.

34. Pankov R, Yamada KM. Fibronectin at a glance. J. Cell Sci. 115(20), 3861-3863 (2002).

35. Williams CM, Engler AJ, Slone RD, Galante LL, Schwarzbauer JE. Fibronectin expression modulates mammary epithelial cell proliferation during acinar differentiation. Cancer Res. 68(9), 3185-3192 (2008).

36. Meena LS, Meena J. Cloning and characterization of a novel PE_PGRS60 protein (Rv3652) of Mycobacterium tuberculosis $\mathrm{H} 37 \mathrm{Rv}$ exhibit fibronectin-binding property. Biotechnol. Appl. Biochem. 63(4), 525-531 (2016).

37. De Oliveira EO, Wang K, Kong HS et al. Effect of the leukotriene A4 hydrolase aminopeptidase augmentor 4-methoxydiphenylmethane in a pre-clinical model of pulmonary emphysema. Bioorg. Med. Chem. Lett. 21(22), 6746-6750 (2011).

38. Xiao Q, Dong N, Yao X et al. Bufexamac ameliorates LPS-induced acute lung injury in mice by targeting LTA4H. Sci. Rep. 6, 25298 (2016).

39. Snelgrove RJ, Jackson PL, Hardison MT et al. A critical role for LTA4H in limiting chronic pulmonary neutrophilic inflammation. Science 330(6000), 90-94 (2010).

40. Okudela K, Mitsui H, Woo T et al. Alterations in cathepsin L expression in lung cancers. Pathol. Int. 66(7), 386-392 (2016).

41. Hamon $\mathrm{Y}$, Legowska $\mathrm{M}$, Herve $\mathrm{V}$ et al. Neutrophilic cathepsin $\mathrm{c}$ is maturated by a multistep proteolytic process and secreted by activated cells during inflammatory lung diseases. J. Biol. Chem. 291(16), 8486-8499 (2016).

42. Steinwede $\mathrm{K}$, Maus R, Bohling J et al. Cathepsin G and neutrophil elastase contribute to lung-protective immunity against mycobacterial infections in mice. J. Immunol. 188(9), 4476-4487 (2012).

43. Rivera-Marrero CA, Stewart J, Shafer WM, Roman J. The down-regulation of cathepsin G in THP-1 monocytes after infection with Mycobacterium tuberculosis is associated with increased intracellular survival of bacilli. Infect. Immun. 72(10), 5712-5721 (2004). 
